\title{
Frequency investigation of rainfall in the sugarcane growing region of North Queensland, Australia
}

\author{
M. Casey and Y. Everingham \\ School of Engineering and Physical Sciences, James Cook University, \\ Townsville, Queensland, 4811, Australia \\ Email: madalyn.casey@my.jcu.edu.au
}

\begin{abstract}
Understanding the underlying patterns of atmospheric systems is an important step in understanding climate variability. The potential impact of climate variability on agricultural industries is an important industry issue. Greater understanding of climate variability on both global and local scales is required. This local scale study analyses the frequency of rainfall in the sugarcane growing region of North Queensland, Australia. Spectral analysis of time series signals allows for the identification of periodic and quasi-periodic frequency components in the temporal structure of climate signals and how these have varied over time. This study used wavelet decomposition to deconstruct the rainfall records for 3 locations in North Queensland: Macknade, Kalamia and Tully to identify the underlying frequency spectrum. Seasonal accumulations of the weather station rainfall records from 1889, 1913 and 1926 to 2008 were computed, and wavelet analysis suggest that North Queensland rainfall at these three locations are influenced by a number of underlying cycles, covering both short and long time periods. A separate analysis of each season across all locations showed similarities between autumn and winter rainfall variability, and summer and spring rainfall. It was shown that the rainfall variability in each season is influenced by both ENSO length periodicities, as well as longer term decadal and interdecadal oscillations. The analysis did not show any changes to how the rainfall signals may be varying, however a quantitative approach may detect such changes. The identification of the periodic cycles influencing the local climate is a useful step towards understanding and controlling local changes in climate variability.
\end{abstract}

Keywords: Frequency Analysis, wavelet Transform, rainfall variability, North Queensland, sugarcane 


\section{INTRODUCTION}

Understanding changes in climate variability has the potential to influence many aspects of human life, from food security and water availability, to resource management and the sustainability of local industries. The potential impacts associated with a changing climate on both global and regional scales emphasise the need for greater understanding of both natural and forced climate variability. At a local level, rainfall variability strongly influences the practices of agricultural industries, where it directly impacts planning of seasonal operations and resource management, and affects crop sizes and quality to influence farm productivity.

Large multi-disciplinary approaches have been undertaken to produce climate models used to understand climate behaviour and attribute its variability to both natural phenomena and anthropogenic, or human induced, changes on the atmospheric-ocean system (Solomon 2007). Global Climate Models may well describe large-scale climate variability, however downscaling of these models does not always provide a good estimate of small-scale variability (Meinke and Stone 2005). While being able to attribute and accurately model variability in the global climate using large scale climate drivers is important, an understanding of variability experienced at smaller, regional scales may facilitate decisions based on more accurate, region-specific climatic conditions.

While many studies have focused on changes to rainfall variability in south-eastern Australia and southeastern Queensland (Speer et al., 2011, Beecham and Chowdhury 2010, Cai et al., 2010, Murphy and Timbal 2008) or over broad regions of Australia (Risbey et al., 2009, Solomon 2007, Walland et al., 2000), little analysis of the rainfall variability at point stations in northern Queensland has been performed. The East coast agricultural region of Australia is unique in its topographical features, resulting in regional variations to large scale climate patterns that are not entirely understood (Park 2008, Power et al., 2006, Risbey et al., 2009, Speer et al., 2011).

The productivity of the Australian sugarcane industry, like many other industries, is heavily influenced by seasonal, inter-annual and longer term variations in rainfall patterns (Park 2008, Nicholls 1985). Planting, harvesting, milling and marketing operations are all directly affected by climate conditions during the relevant seasons (Park 2008, Everingham et al., 2002, Jones and Everingham 2005). Differences in the variability of climate conditions between seasons has been identified in a number of regions (Nicholls 2006, Solomon 2007). Because industry decisions are often based on seasonal rainfall conditions, season-specific investigations are of particular interest.

This study identifies the temporal characteristics of seasonal rainfall variability for three sugarcane growing locations in northern Queensland, with the aim of providing a greater understanding of the rainfall patterns experienced in these regions. Advanced knowledge of the seasonal and regional variations in climate conditions may assist industry decision makers to exploit or mitigate the impacts of changing climatic conditions.

\section{DATA AND METHODS}

\subsection{Data}

Daily rainfall data for three locations in the north-eastern Queensland sugar cane region were sourced from the Australian Bureau of Meteorology. Two of the three locations, Macknade (station number 32032), located at $18^{\circ} 36^{\prime} \mathrm{S}$ and $146^{\circ} 15^{\prime} \mathrm{E}$, and Kalamia (station number 33035 ), located at $19^{\circ} 31^{\prime} \mathrm{S}$ and $147^{\circ} 25^{\prime} \mathrm{E}$, are part of the Australian high quality climate site network (Lavèry et al., 1992, Jeffrey et al., 2001). The third location, Tully (station number 32042 ), located at $17^{\circ} 56^{\prime} \mathrm{S}$ and $145^{\circ} 55^{\prime} \mathrm{E}$, is not part of the high quality network and may contain errors and biases not corrected for.

For each location, the selected time period represents the longest part of the record where observations are mostly directly recorded as daily rainfall totals. Macknade data from 1899 to 2008 contains $87.3 \%$ of observations directly recorded for each day, while the Kalamia record from 1913 to 2008 contains $97.9 \%$ exact observations, and the Tully record from 1926 to 2008 contains $98.5 \%$ of observations directly recorded for each day. The reader is referred to (Lavèry et al., 1992, Jeffrey et al., 2001) for a description of the methods used to assign observations for days when exact data was not recorded.

For each station, the total rainfall accumulation for each calendar season was computed, producing a summer rainfall total that is the sum of rainfall during December, January and February, and so on for each other season. The accumulated rainfall for each season was analysed separately to assess whether changes in the variability could be detected between seasons. 


\subsection{Methods}

\section{Wavelet Theory}

Wavelet decomposition is a spectral analysis technique that decomposes a time series signal in terms of similarly shaped, or scaled, versions of a particular analysing function, a finite wave called a mother wavelet. Instead of decomposing a signal into infinite sine and cosine components as in Fourier spectral analysis, the wavelet transformation computes coefficients that are a measure of the correlation between the signal being analysed, and translations and dilations, or scales, of the mother wavelet. The continuous wavelet transform (CWT) of a function of the square-integrable class $L^{\mathbf{2}}(\mathbf{R})$, is given by the integral of the convolution of a set of wavelet functions with the continuous data sequence over the set of real numbers, $\mathbf{R}$ (Farge 1992). For a discretely sampled finite length signal, $f(t)$, where $t=0, \ldots, N-1$ and $N$ is the number of samples, with equal time spacing, $\delta t$, the CWT takes the form

$$
W_{\psi}(a, b)=\frac{1}{\sqrt{\alpha}} \sum_{t=-\infty}^{\infty} f(t) \psi \cdot\left(\frac{t-b}{a}\right)
$$

where * represents the complex conjugate, and $\psi$ is a 'daughter' wavelet, a translated by $b$ and dilated or scaled by $a$, form of the mother wavelet (Torrence and Compo 1998, Farge 1992). The inclusion of the $a^{-1 / 2}$ term in (1) directly relates the estimated wavelet spectrum to the true wavelet spectrum, however also overemphasises the power of coefficients at large scales (Schaefli et al., 2007).

The use of all possible scales (parameter $a$ ) and translations (parameter $b$ ) of the mother wavelet results in an over-complete set of wavelet coefficients (Kestin et al., 1998, Meyers et al., 1993, Farge 1992). The resulting redundancy in these coefficients means coefficients of neighbouring scales and time points are correlated, complicating the reconstruction of the original signal (Torrence and Compo 1998, Schaefli et al., 2007, Farge 1992). The discrete wavelet transform (DWT) uses a much sparser set of scale and dilation parameters to form an orthogonal basis of daughter wavelets, resulting in a more efficient analysis well suited to signal compression and reconstruction (Lau and Weng 1995, Farge 1992, Meyers et al., 1993). For the current application, however, an analysis with good time-scale localisation is preferable for identifying the signal's underlying frequency content, so the wavelet decomposition is performed $\mathrm{N}$ times at each scale, where $\mathrm{N}$ is the length of the signal, to approximate a CWT (Torrence and Compo 1998, Kaiser 1994).

When approximating a CWT, a discrete sample of scale and dilation parameters must be used, the choice of which is arbitrary when a non-orthogonal decomposition is performed (Torrence and Compo 1998). The choice of scales are often expressed as fractional powers of two, and depend on both the time spacing of the signal, $\delta t$, and the scale step size for the analysis, $\delta j$. The discrete scales are given by $a_{j}=a_{0} 2 \delta j$, where $l=0,1_{1, \ldots} l$, the total number of scales is given by $l=\delta f^{-1} \log _{2}\left(\frac{N \delta t}{a_{0}}\right)$, and the smallest resolvable scale, $a_{0}$, may be given by $20 t$ (Torrence and Compo 1998, Weng and Lau 1994). For the current analysis, $\delta t=1$ sample per year, $u_{0}=2,0 f$ was chosen as 0.001 to give fine scale resolution, and $I$ becomes $0.001^{-1} \log _{2}\left(\frac{N}{2}\right)$, for example, the total number of scales is $7=\mathbf{5 8 5 4}$.

The only constraint placed on the analysing function for it to be considered a mother wavelet is the admissibility condition (Farge 1992). The condition requires that the function has finite energy and oscillates with a zero mean (Farge 1992, Weng and Lau 1994). Other conditions may be placed on the analysing function to give attributes that are useful in a variety of applications. For the current application, a non-orthogonal mother wavelet provides good time-scale localisation to facilitate the identification of a signal's underlying spectral components (Torrence and Compo 1998, Kaiser 1994). While real valued wavelets can isolate peaks and discontinuities in a signal, a complex mother wavelet is useful for identifying oscillatory behaviour in a series (Torrence and Compo 1998). Since the shape of the mother wavelet should be similar to the features of the signal being analysed, a wavelet with a sinusoidal shape assists in the identification of some of the oscillatory components of climate signals which are almost sinusoidal in shape, such as annual cycles and approximately 2-7 year El Niño-Southern Oscillation (ENSO) signals (Gu and Philander 1995). Mother wavelets also have other properties not discussed here, 
such as compact support, symmetry and number of vanishing moments, each useful for other analysis types, see Farge (1992), Kaiser (1994), Torrence and Compo (1998), and Walczak (2000).

The complex Morlet wavelet, a plane wave modulated by a Gaussian envelope of unit width, is commonly used in climate applications as its complex nature and sinusoidal shape allows for the identification of some of the cyclic components of climate signals (Beecham and Chowdhury 2010, Kirkup et al., 2001, Nakken 1999, Souza Echer et al., 2008, Torrence and Compo 1998, Speer et al., 2011, Weng and Lau 1994, Lau and Weng 1995, Schaefli et al., 2007, Maraun and Kurths 2004). The Morlet wavelet, given by (2), does not form an orthogonal basis so an arbitrary set of scales may be used to increase the time-scale resolution of the decomposition.

$$
\psi(t)=s^{-i \alpha_{\mathrm{c}} \varepsilon_{e}}-1 \mathrm{t}^{2} / \mathrm{s}
$$$$
\omega_{2}=\pi \sqrt{\frac{2}{\ln 2}} \approx 5.336
$$
where the non-dimensional frequency to satisfy the admissibility condition
(Farge 1992, Strang and Nguyen 1996, Lau and Weng 1995). It should be noted that the Morlet wavelet is only "marginally admissible" and does not have a zero mean without the addition of some very small correction terms, which become negligible if $\omega_{\mathbf{0}}=\mathbf{0}$ is used (Farge 1992).
\end{abstract}

\title{
Implementation
}

In the computation of the wavelet coefficients, the convolution product can be performed as in (1), or the convolution theorem can be utilised to reduce this product to a simple multiplication in the frequency domain, thereby reducing computational time (Farge 1992). In the frequency domain, a more efficient algorithm, the fast Fourier Transform (FFT) may be used to further reduce computation time. For further details, the reader is referred to Torrence and Compo (1998). Since the wavelet transform assumes the data sequence is continuous and cyclic, rather than finite in length, errors at the beginning and end of each analysis are produced (Torrence and Compo 1998). Expanding the signal with zeros reduces the number of wavelet coefficients of the original data influenced by these edge effects, but also results in the inclusion of these zeros in the analysis at larger scales. The coefficients corresponding to the zero-padding are removed after analysis, and a 'cone of influence' is defined as the region of the spectrum where coefficients are still affected by these discontinuous-edge effects (Torrence and Compo 1998, Lau and Weng 1995).

\section{Power Spectrum}

The wavelet coefficients generated by wavelet decomposition using the complex Morlet wavelet are also complex valued. The wavelet power spectrum can be defined as the square of the amplitude of the wavelet coefficients, $\left|U_{\psi}^{*}\left(\sigma_{0} b\right)\right|^{3}$. High power values represent a high contribution of the particular scale to the oscillatory variability in the rainfall signal at the particular time, while low power values represent a small contribution of the given scale to the variability in the rainfall signal at the particular time. To ensure the transform at different scales can be compared, and the wavelet transform can be compared to other time series analysis methods, the wavelet transform is normalised at each scale to have unit energy (Torrence and Compo 1998, Farge 1992).

\section{RESULTS AND DISCUSSION}

The wavelet power spectra for each of the different stations for some seasons are shown in Figures 1 to 4. The colour range of each plot displays the lowest power periods of oscillation as blue and the highest power periods of oscillation as dark red. Each figure also displays a time-series plot of the rainfall accumulation values used to generate the power spectrum, and the cone of influence for the wavelet coefficients, shown as a thin black line.
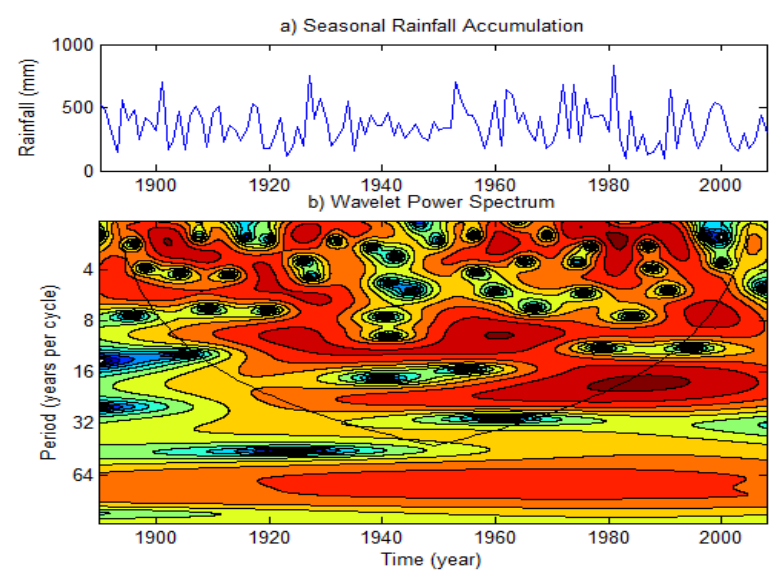

Figure 1. (a) Annual summer rainfall accumulation values from 1899 to 2008 for Macknade, (b) wavelet power spectrum. 
For the analysis of summer data, all three locations exhibit strong periodicity in rainfall variability in the range of 16-32 year length cycles from approximately 1950 onwards, e.g. see Macknade location (Figure 1). Data for Kalamia and Tully summer analyses are not shown. From approximately 1950 onwards all sites show high values for the coefficients associated with 2-8 year periodicity. All three stations also show low contribution to rainfall variability in the range of 2-10 year cycles around the 1940s.

For the analysis of autumn data, all three locations have strong wavelet coefficients in the 2-8 year periodicity range between the 1940s and 1950s, e.g. see Kalamia location (Figure 2). Data for Macknade and Tully autumn analyses are not shown. Longer length cycles, up to 16 year periodicities, in rainfall variability in the range of 16-32 year length cycles from approximately 1950 onwards, see Figure 2. From approximately 1950 onwards all sites show high values for the coefficients associated with 2-8 year periodicity. All three stations also show low contribution to rainfall variability in the range of 2-10 year cycles around the 1940s.

The winter rainfall accumulation data shows periodicities similar in power to those in the autumn data analysis, however, rainfall variability appears to also be influenced by cycles $16-20$ years in length at Macknade and Kalamia. Tully, however, does not exhibit this same pattern of interdecadal oscillation affecting winter rainfall, see Figure 3. Data for Macknade and Kalamia winter analyses are not shown.

Spring rainfall for all locations shows similar patterns to the summer conditions, with long term cycles having a high influence on regional rainfall variability. Figure 4 shows the spring rainfall analysis for Tully, with data for Macknade and Kalamia not shown.

For the analysis of seasonal data for each of the three locations, all stations showed 2-8 year periodicities have a strong influence on rainfall variability. This is not unexpected since it is known that the ENSO influences north-eastern Australian rainfall on a time scale between 2 and 7 years. Another feature in each analysis (except Tully winter rainfall) showed that longer cyclic components have similar
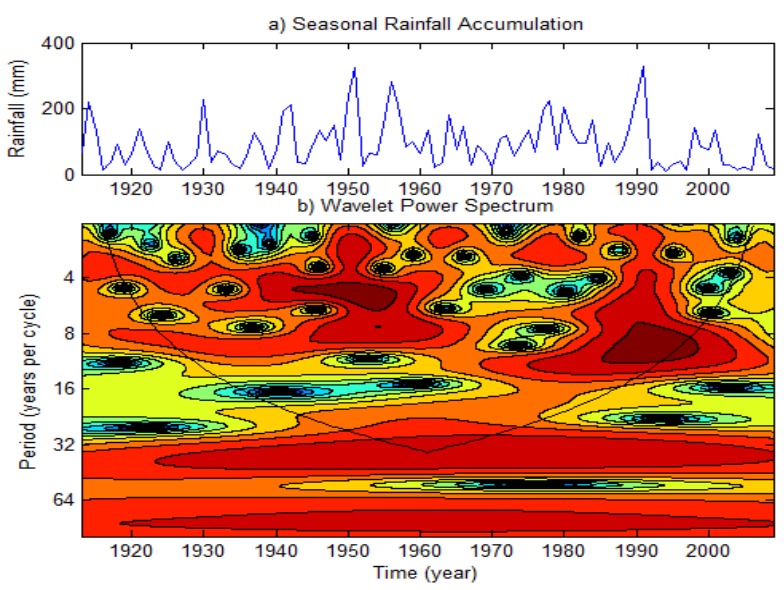

Figure 2. (a) Annual autumn rainfall accumulation values from 1913 to 2008 for Kalamia, (b) wavelet power spectrum.
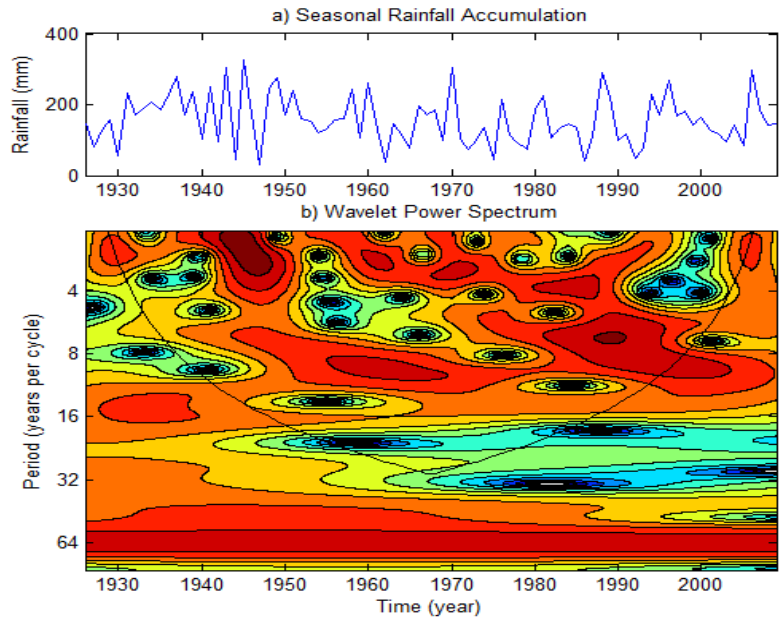

Figure 3. (a) Annual winter rainfall accumulation values from 1926 to 2008 for Tully, (b) wavelet power spectrum.
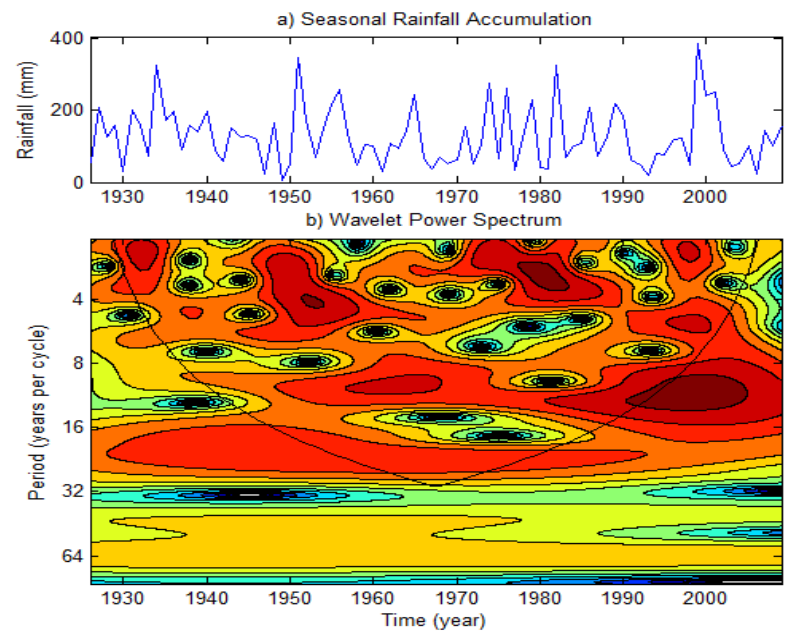

Figure 4. (a) Annual spring rainfall accumulation values from 1926 to 2008 for Tully, (b) wavelet power spectrum. 
power values to cycles in the ENSO range. This result suggests that interdecadal oscillations contribute to North Queensland rainfall variability as strongly as ENSO cycles do. However, this result may not be true due to the $L^{\mathbf{2}}$ normalisation of coefficients overestimating powers at large scales. The correlation between neighbouring coefficients may result in areas of 'false positives' where high power coefficients may not be a feature of the true wavelet spectrum.

A qualitative assessment of whether the rainfall variability itself may be changing over time does not appear to show that this is the case for the seasonal accumulation data analysed here. With the correlation and overestimation problems affecting the analysis, possibly obscuring these changes, a quantitative analysis may be preferable for detecting changes in rainfall variability.

\section{CONCLUSION}

The qualitative approach used to assess the underlying cyclic nature of rainfall variability in North Queensland shows that periodicities in the range of ENSO cycles, between 2 and 7 years, strongly influence rainfall during all seasons. The analysis also shows that longer term cycles, on decadal and interdecadal time scales also affect rainfall variability, often with similar power to the dominant ENSO cycles. To overcome some of the problems associated with the wavelet analysis, a quantitative approach may distinguish between periods of strong influence and cycles that are statistically significant. A quantitative assessment of whether climate variability is changing over time would be useful for understanding current climate conditions and incorporating this knowledge into industry practices.

\section{ACKNOWLEDGEMENTS}

This research has been supported through funding provided by the Australian Government through the Sugar Research and Development Corporation and by James Cook University.

\section{REFERENCES}

Beecham, S. and R. K. Chowdhury (2010). Temporal characteristics and variability of point rainfall: a statistical and wavelet analysis. International Journal of Climatology, 30, 458-473.

Cai, W., P. van Rensch, T. Cowan and A. Sullivan (2010). Asymmetry in ENSO Teleconnection with Regional Rainfall, Its Multidecadal Variability, and Impact. Journal of Climate, 23, 4944-4955.

Everingham, Y. L., R. Muchow, R. C. Stone, N. Inman-Bamber, A. Singels and C. Bezuidenhout (2002). Enhanced risk management and decision-making capability across the sugarcane industry value chain based on seasonal climate forecasts. Agricultural Systems, 74, 459-477.

Farge, M. (1992). Wavelet transforms and their applications to turbulence. Annual Review of Fluid Mechanics, 24, 395-457.

Gu, D. and S. Philander (1995). Secular changes of annual and interannual variability in the Tropics during the past century. Journal of Climate, 8, 864-876.

Jeffrey, S. J., J. O. Carter, K. B. Moodie and A. R. Beswick (2001). Using spatial interpolation to construct a comprehensive archive of Australian climate data. Environmental Modelling and Software, 16, 309330 .

Jones, K. and Y. Everingham (2005). Can ENSO combined with Low-Frequency SST signals enhance or suppress rainfall in Australian sugar growing areas. In: MODSIM 2005 International Congress on Modelling and Simulation, edited by: Zerger, A. and Argent, R. M., Melbourne, December, Modelling and Simulation Society of Australia and New Zealand, 1660-1666.

Kaiser, G. (1994). A friendly guide to wavelets. Birkhauser.

Kestin, T. S., D. J. Karoly, N. A. Rayner and J.-I. Yano (1998). Time-Frequency Variability of ENSO and Stochastic Simulations. Journal of Climate, 11, 2258-2272.

Kirkup, H., A. Pitman, J. Hogan and G. Brierley (2001). An initial analysis of river discharge and rainfall in coastal New South Wales, Australia using wavelet transforms. Australian Geographical Studies, 39, 313-334.

Lau, K. M. and H. Weng (1995). Climate Signal Detection Using Wavelet Transform: How to Make a Time Series Sing. Bulletin of the American Meteorological Society, 76, 2391-2402.

Lavèry, B., A. Kariko and N. Nicholls (1992). A historical rainfall data set for Australia. Australian Meteorological Magazine, 40, 7.

Maraun, D. and J. Kurths (2004). Cross wavelet analysis: significance testing and pitfalls. Nonlinear Processes in Geophysics, 11, 505-514.

Meinke, H. and R. C. Stone (2005). Seasonal and inter-annual climate forecasting: the new tool for increasing preparedness to climate variability and change in agricultural planning and operations. Increasing Climate Variability and Change, 221-253. 
Meyers, S. D., B. G. Kelly and J. J. O'Brien (1993). An introduction to wavelet analysis in oceanography and meteorology - with application to the dispersion of Yanai waves. Monthly Weather Review, 121, 2858-2866.

Murphy, B. F. and B. Timbal (2008). A review of recent climate variability and climate change in southeastern Australia. International journal of Climatology, 28, 859-879.

Nakken, M. (1999). Wavelet analysis of rainfall-runoff variability isolating climatic from anthropogenic patterns. Environmental Modelling and Software, 14, 283-295.

Nicholls, N. (1985). Impact of the Southern Oscillation on Australian crops. International Journal of Climatology, 5, 553-560.

--- (2006). Detecting and attributing Australian climate change: a review. Australian Meteorological Magazine, 55, 199-211.

Park, S. (2008). A review of climate change impact and adaptation assessments on the Australian sugarcane industry. Australian Society of Sugar Cane Technologists, 1-9.

Power, S., M. Haylock, R. Colman and X. Wang (2006). The predictability of interdecadal changes in ENSO activity and ENSO teleconnections. Journal of Climate, 19, 4755-4771.

Risbey, J. S., M. J. Pook, P. C. McIntosh, M. C. Wheeler and H. H. Hendon (2009). On the remote drivers of rainfall variability in Australia. Monthly Weather Review, 137, 3233-3253.

Schaefli, B., D. Maraun and M. Holschneider (2007). What drives high flow events in the Swiss Alps? Recent developments in wavelet spectral analysis and their application to hydrology. Advances in Water Resources, 30, 2511-2525.

Solomon, S. (2007). Climate Change 2007: the physical science basis: contribution of Working Group I to the Fourth Assessment Report of the Intergovernmental Panel on Climate Change. Cambridge Univ Pr.

Souza Echer, M., E. Echer, D. Nordemann, N. Rigozo and A. Prestes (2008). Wavelet analysis of a centennial (1895-1994) southern Brazil rainfall series (Pelotas, $31^{\circ} 4619 \mathrm{~S} 52^{\circ} 2033 \mathrm{~W}$ ). Climatic Change, 87, 489-497.

Speer, M. S., A. O. Fierro and L. M. Leslie (2011). Australian east coast rainfall decline related to large scale climate drivers. Climate Dynamics, 36, 1419-1429.

Strang, G. and T. Nguyen (1996). Wavelets and filter banks. Wellesley Cambridge Pr.

Torrence, C. and G. P. Compo (1998). A Practical Guide to Wavelet Analysis. Bulletin of the American Meteorological Society, 79, 61-78.

Torrence, C. and P. J. Webster (1999). Interdecadal changes in the ENSO-monsoon system. Journal of Climate, 12, 2679-2690.

Walczak, B. (Ed.) (2000). Wavelets in chemistry, pp.76-78. Elsevier, Amsterdam.

Walland, D., S. Power and A. Hirst (2000). Decadal climate variability simulated in a coupled general circulation model. Climate dynamics, 16, 201-211.

Weng, H. and K. M. Lau (1994). Wavelets, Period Doubling, and Time-Frequency Localization with Application to Organization of Convection over the Tropical Western Pacific. Journal of the Atmospheric Sciences, 51, 2523-2541. 